\title{
CENSORSHIP OF LEAKAGE PARAMETERS OF A FINFET BASED SCHMITT TRIGGER AT NANO-METER REGIME \\ Vishwas Mishra $^{1 *}$, Abhishek Kumar ${ }^{2}$, Shobhit Tyagi ${ }^{3}$, Neha Verma ${ }^{4}$, Divya Mishra ${ }^{5}$ \\ $1^{*}, 2,3,4,5$ Assistant Professor, Swami Vivekananda Subharti University, Meerut, India. \\ Email: 1*vishwasmishra88@gmail.com, ${ }^{2}$ abhishekec02@gmail.com, ${ }^{3}$ shobhittyagi.1857@gmail.com, \\ 4nehav70@gmail.com, 5 mishradivya1311@gmail.com
}

Article History: Received on $12^{\text {th }}$ April 2020, Revised on $20^{\text {th }}$ May 2020, Published on $16^{\text {th }}$ June 2020

\begin{abstract}
Purpose: Recently FinFET technology has gained a lot of attention because of its superior fabrication process that is very similar to the fabrication of a conventional transistor. FinFETs unique feature as well as the potential applications make it a strong contender for the low power chip designs. Research is in full swing to use FinFET in analog circuits like Schmitt trigger, sensors, OPAMP and digital logic. The realization of the FinFET based circuits predicts that it is possible to broaden the concept of Moore's law without unstoppable scaling of CMOS devices.
\end{abstract}

Methodology: This work is carried out on the Candence Simulation tool. After the simulation, all these parameters have been compared with previous published $4 \mathrm{~T}$ Schmitt trigger at $45 \mathrm{~nm}$ with this design and found that they are in close vicinity.

Main Findings: By combining the superior flexibility and reduced short channel effects (SCEs) of FinFET devices offers a promising approach to implement highly integrated, power-efficient Schmitt Trigger circuit for low power digital applications. Schmitt trigger is a device capable of removing unwanted noise from the input and prevent the other operations from this unwanted noise and improve the performance of the device.

Implications: This study is discussing and performs a comparative analysis of different leakage parameters of a FinFET based Schmitt Trigger with previous 4T Schmitt Trigger at $45 \mathrm{~nm}$.

The novelty of Study: Size, power, speed, Cost etc. are important factors for designing any new circuits in the field of Electronics. Various eminent researchers have been making efforts for this. This paper makes some effort to discuss about past research and design a new circuit where the value of delay, leakage power and dynamic power reduces when compared to previously published circuits.

Keywords: Schmitt Trigger, Slew Rate, Leakage Power, Average Power, Delay.

\section{INTRODUCTION}

The optimization of power has become very necessary due to the increased number of transistors per unit area and the higher portability demands of the user. Power Dissipation in VLSI circuits is mainly arisen due to three major sources: power required to charge or discharge a node, power due to the output transition and power due to leakage current. Various techniques were devised by the designers for the minimization of power in circuits. Sleep Transistor approach, Multi-Threshold CMOS, SVL, AVL and Hybrid techniques are remarkable for the reduction of leakage currents, which constitutes a major fraction of power in the nano-regime (Steyaert, M. \& Sansen, W. (1986)). The sizing of the transistor is also helpful for minimizing power. By lowering the supply voltages (voltage scaling), power can be saved but the system works slower. In 1965 Gordon Moore published a chapter that expresses the brightest future of integrated technology. Only after 6 years, first monolithic IC was invented, As Moore already mention that IC technology evolves the cost of the transistor decreases steadily that means that a large number of the transistor are going to fitted onto a single silicon chip. Short channel devices are those devices which use the transistors with channel lengths less than 3 to 5micron. Short channel devices comprehensively reduce the ratio between lateral and vertical dimensions (Pfister, A. (1992)). The effects of the transition region from the drain the source diffusions to the channel become significant, causing the need for increased complexity in the device model. The rest of the manuscript is arranged as given. In section II, presents about the description of Schmitt trigger and their characteristics with simulation results. Finally, the conclusion and directions for the future scope of research are given in Section III.

\section{SCHMITT TRIGGER}

Traditional Schmitt Trigger is configured which is an operational amplifier along with resistance where it forms a positive feedback network that the reason traditional Schmitt Trigger design is suited for the complementary-MOS technology implementation, due to the presence of feedback, circuit output accuracy is weakened, makes wastage of die area and give a small amount of sheet resistance. This is the reason where various conventional CMOS designed Schmitt trigger circuits have emerges in a few years. Traditional Schmitt Triggers have large gain for an operational amplifier so it consumes many areas of the chip (Wang, Z. (1991)). MOSFET from the last few years scaled-down largely which is giving birth to the short channel effect. IC performance now depends on the dimensions of the scaling factor so the improvement of such force is important. Figure 1 is a diagram of high-speed Schmitt trigger with 5 transistors. 


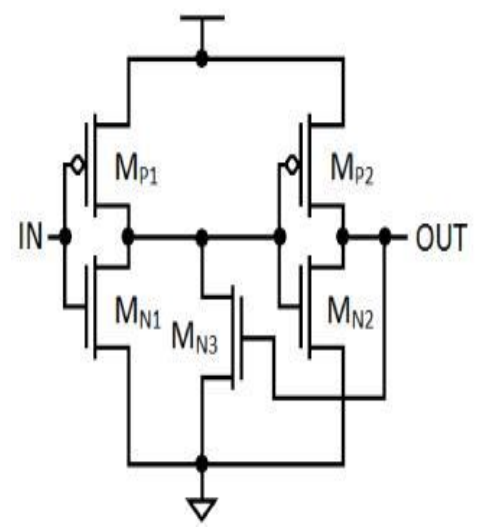

Figure 1: 5T high speed Schmitt Trigger

Now as we scaled down the channel length of the MOSFET some major concerns are arising such as velocity saturation, gate leakage, short channel effects, sub-threshold voltage, mobility degradation and leakage current at below 90nm technology. All these reliability problems are overcome in the new device and also in new material as power consumption increases the heat dissipation is also increases so we required the new device and its application which are used in the VLSI circuits. Moore's law is now not applicable because of the fast-developing technology of VLSI design industries so the conventional devices are replaced by the newly discovered devices and the upcoming devices are target to apply Moore's law in the circuit, FinFET is used as a new device which is work below $22 \mathrm{~nm}$ technology also and its power consumption is also low due to which heat generation is less in the circuit with good mechanical, electrical and thermal properties. FinFET is used in various places such as where we use CMOS now we can use the FinFET circuit it provides great electrical property in the circuit and provides great electrostatic control in the circuit at the gate which provides high driving current (Dokic, B.L. (1984)).

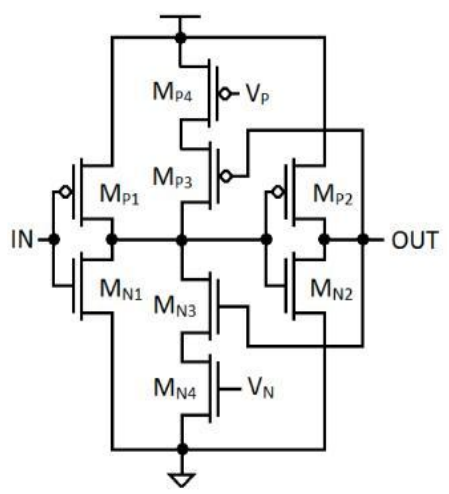

Figure 2: Show the 8T Schmitt trigger

Various complementary-MOS Schmitt Trigger designs have been discovered in the last few years and design is based on the application which we required in the field. Various Schmitt Trigger circuits are studied in the chapter and compared with each other. $\mathrm{M}_{\mathrm{P} 1}-\mathrm{M}_{\mathrm{N} 1}$ and $\mathrm{M}_{\mathrm{P} 2}-\mathrm{M}_{\mathrm{N} 2}$ are the two basic inverters present in the 5T Schmitt Trigger and feedback are provided by the additional nMOS $\left(\mathrm{M}_{\mathrm{N} 3}\right)$ transistor as shown in Figure below hysteresis characteristic in the circuit is controlled by the $\mathrm{M}_{\mathrm{N} 3}$ transistor and the width of the hysteresis characteristic is monitored and varied by feedback transistor.

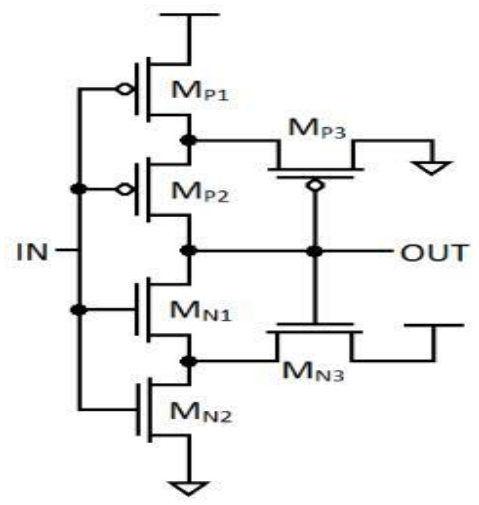

Figure 3: Show the 6T Inverting Conventional Schmitt Trigger 
In this design, we get a higher witching speed. nMOS and pMOS are the two inverter amplifiers with one basic inverting amplifier and there are three pairs of inverters that given in figure 3, this comprises the 6T Schmitt trigger. In wireless communication, the main requirement id the low power consumption but 6T Schmitt trigger have four transistors in its so it consumes more power so it is not used in the low power circuit. However, the 4T Schmitt trigger (Weste, N. \& Eshraghian, K. (1993)) is designed by using a combination of one pMOS (Pi) and three nMOS (Ni, N2 and N3) at 45nm CMOS technology node, this circuit does not have a direct connection from voltage supply to the ground node. Hence it provides the advantage of no static power dissipation. Table 1describes the parameter like leakage power and delay of the 4T Schmitt trigger. In this Schmitt trigger the hysteresis characteristics control by the electrically controlled signal know as $V_{P}$ and $V_{N}$ that permit the hysteresis of the circuit is controlled by the signals which is the use of off-chip potentiometers. As $\mathrm{M}_{\mathrm{P} 3}-\mathrm{M}_{\mathrm{P} 4}$ and $\mathrm{M}_{\mathrm{N} 3}-\mathrm{M}_{\mathrm{N} 4}$ transistor resistor of the circuit is varied with $\mathrm{V}_{\mathrm{P}}$ and $\mathrm{V}_{\mathrm{N}}$ of the circuit respectively (Pedroni, V. A. (2005)).

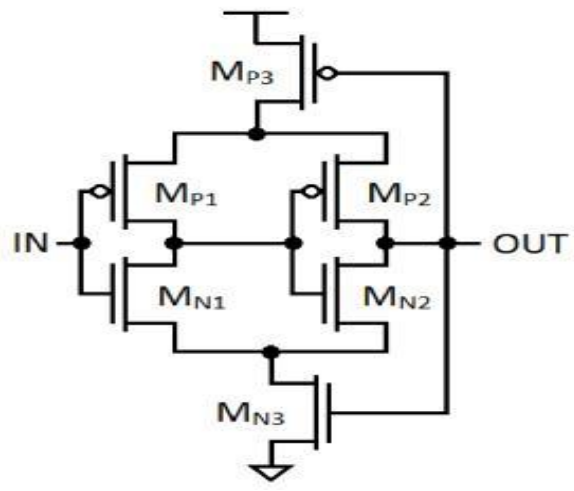

Figure 4: 6T low power Schmitt trigger

In this process, the threshold voltage of the circuit is adjusted. Due to this process, the static power dissipation of the circuit is decreased. Different characteristics and their results are described below.

\section{a. Slew rate}

The design of the N-type and P-type transistor very crucial because due it there is a short time when both P-type and Ntype transistor is switch on at the same time at the transition of either 0 to 1 or 1 to 0 , this gives the current short pulse from to $\mathrm{V}_{\text {ss }}$ for a very short time (Saxena, A. et al., (2014)).

Table 1: Slew rate of the FinFET based Schmitt trigger

\begin{tabular}{ll}
\hline $\mathbf{V}_{\text {dd }}$ (volts) & $\begin{array}{l}\text { Slew } \\
\text { RateRgbrvRRater } \\
\text { ate(volts/pSec) }\end{array}$ \\
\hline 0.6 & 5.88 \\
\hline 0.7 & 6.864 \\
\hline 0.8 & 4.978 \\
\hline 0.9 & 3.727 \\
\hline
\end{tabular}

sFrom table 1, we can observe that it achieves a maximum slew rate of 6.864 volts/psec for a time $269.6 \mathrm{pSec}$ at 0.7 volts. Hence at 0.7 volts change its output rapidly.

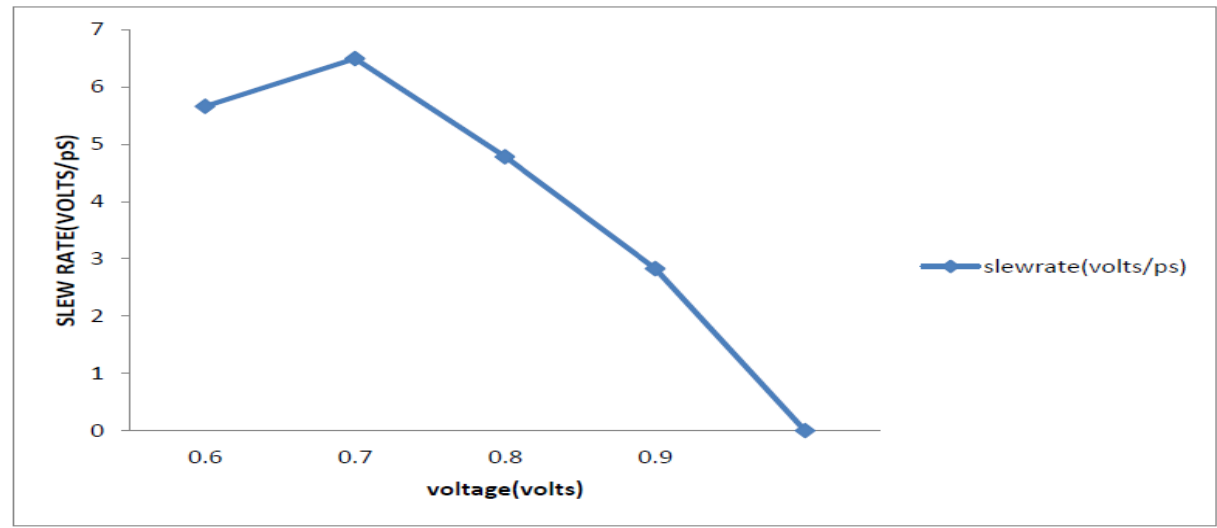

Figure 5: Shows the Slew Rate of 4T Schmitt Trigger 
Figure 5 elaborates that there is a reduction in delay of FinFET based Schmitt trigger $37.38 \%$ at 0.7 volts power supply from 4T Schmitt trigger.

Table 2: 4T Schmitt trigger parameters

\begin{tabular}{lll}
\hline $\begin{array}{l}\text { Supply } \\
\text { Voltage }\end{array}$ & Leakage power(pW) Delay $(\mathbf{p S e c})$ \\
\hline 0.6 & 2.54 & 540.3 \\
\hline 0.7 & 4.86 & 485.4 \\
\hline 0.8 & 13.98 & 409.78 \\
\hline 0.9 & 14.34 & 389.29 \\
\hline
\end{tabular}

Table 2 describes the characteristics i.e. leakage power and delay of 4T Schmitt trigger at different voltages. In this Schmitt trigger we allowing the $B_{p}$ and $B_{n}$ that is trans-conductance parameters are same then the width of the hysteresis (Mishra, V. \& Akashe, S. (2015)) of the circuit becomes independent of the threshold voltage and now the hysteresis is depended the only o the supply voltage and the geometry of the circuit. In the $6 \mathrm{~T}$ low power Schmitt trigger, there are three transistors incorporated with the CMOS design as shown in figure 6.

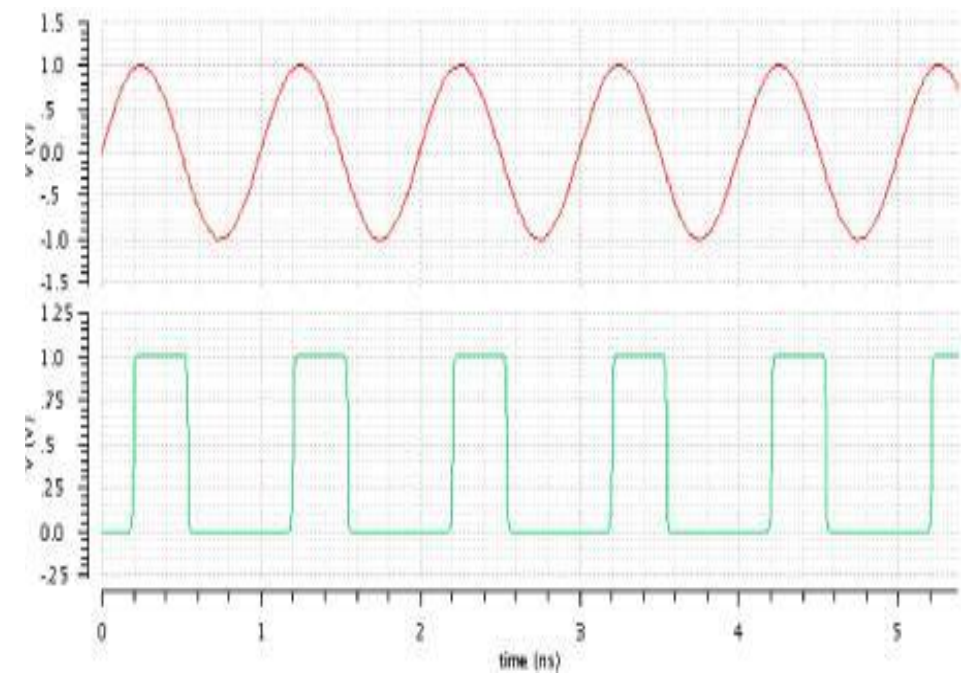

Figure 6: FinFET Schmitt trigger input-output waveform

\section{b. Delay}

Delay is termed as the fifty present input and fifty present of output time taken by the signal is called the delay of the circuit. Delay can be also termed as the time required by the signal to propagate from input to output node (Mishra, V. \& Akashe, S. (2014)).

Delay $=0.69 R_{\text {eq }} \times$ CL (1)

Where equivalent resistance of the design is given by $R_{e q}$ and the load capacitance is given by CL. FinFET based Schmitt trigger reduces the delay by $21.26 \%$ at 0.7 volts power supply from 4T Schmitt trigger (Al-Sarawi, S. F. (2002))

\section{c. Power analyses}

The energy consumption per unit time is known as the amount of power dissipated in the circuit. Charging and discharging of the load capacitor gives rise to the dynamic power dissipation. The transient switching current is also coming in the picture in this charging and discharging of the capacitor (Khandelwal, S. et al., (2013)). The design of the $\mathrm{N}$-type and P-type transistor very crucial because due it there is a short time when both P-type and N-type transistor is switch on at the same time at the transition of either 0 to 1 or 1 to 0 , this gives the current short pulse from $V_{d d}$ to $V_{s s}$ for a very short time.

\section{d. Dynamic \& leakage power of schmitt}

The energy consumption per unit time is known as the amount of power dissipated in the circuit. Charging and discharging of the load capacitor gives rise to the dynamic power dissipation (Katyal, V. et al., (2008)). The transient switching current is also come in the picture in this charging and discharging of the capacitor. The design of the N-type 
and P-type transistor very crucial because due it there is a short time when both P-type and N-type transistor is switch on at the same time at the transition of either 0 to 1 or 1 to 0 , this gives the current short pulse from $V_{\text {dd }}$ to $V_{s s}$ for a very short time (Saini, S. et al., (2009)). Table 3 shows the Average Power and Leakage Power of 4T Schmitt Trigger.

Table 3: Shows the Average Power and Leakage Power of 4T Schmitt Trigger

\begin{tabular}{lllll}
\hline $\mathbf{V}_{\text {dd }}($ volts) & 0.6 & 0.7 & 0.8 & 0.9 \\
\hline $\begin{array}{l}\text { Average } \\
\text { power(pW) }\end{array}$ & 6.45 & 10.258 & 1.3458 & 17.236 \\
$\begin{array}{l}\text { Leakage } \\
\text { Power(pW) }\end{array}$ & 2.256 & 2.359 & 2.456 & 3.001 \\
\hline
\end{tabular}

\section{CONCLUSION}

FinFETs unique feature as well as the potential applications make it a strong contender for the low power chip designs. Research is in full swing to use FinFET in analog circuits like Schmitt trigger, sensors, OPssAMP and digital logic. The realization of the FinFET based circuits predicts that it is possible to broaden the concept of Moore's law without unstoppable scaling of CMOS devices. By combining the superior flexibility and reduced short channel effects (SCEs) of FinFET devices offers a promising approach to implement highly integrated, power-efficient Schmitt Trigger circuit for low power digital applications. After the simulation, all these parameters have been compared with previous published $4 \mathrm{~T}$ Schmitt trigger at $45 \mathrm{~nm}$ with this design and found that they are in close vicinity. Our design reduces delay, leakage power and dynamic power.

\section{ACKNOWLEDGEMENT}

This work is carried out on the Candence Simulation tool. So, we all are thankful for Swami Vivekananda Subharti University, Meerut.

\section{REFERENCES}

1. Al-Sarawi, S. F. (2002). Low-power Schmitt trigger circuit. Electron. Letter. 38, 1009-1010. https://doi.org/10.1049/el:20020687

2. Dokic, B.L. (1984). CMOS Schmitt triggers. IEEE Electronic Circuits System, 131(5), 197- 202. https://doi.org/10.1049/ip-g-1.1984.0037

3. Katyal, V., Geiger, R. L. \& Chen, D. J. (2008). Adjustable Hysteresis CMOS Schmitt Trigger. IEEE International Symposium on Circuits and system, ISCAS, 1938-1941.

4. Khandelwal, S., Raj, B. \& Gupta, R.D. (2013). Leakage Current and Dynamic Power Analysis Of Finfet Based 7T SRAM at 45nm Technology. International Arab Conference on Information Technology (ACIT'2013), Sudan University.

5. Mishra, V. \& Akashe, S. (2014). Calculation of Power Delay Product and Energy Delay Product in 4-Bit FinFET Based Priority Encoder. Springer Proceedings, International Conference on Opto-Electronics and Applied Optics (IEM OPTRONIX-2014), Kolkata, India, pp. 283-289. https://doi.org/10.1007/978-81-3222367-2_36

6. Mishra, V. \& Akashe, S. (2015). Calculation of Average Power, Leakage power, and leakage Current of FinFET based 4-bit Priority Encoder. Proceeding of Fifth IEEE International Conference on Advance Computing \& Communication Technologies (ACCT), pp. 65-69, 21 Feb 2015, Rohtak, Haryana. https://doi.org/10.1109/ACCT.2015.82

7. Pedroni, V. A. (2005). Low-voltage high-speed Schmitt trigger and compact window comparator. Electron. Letter, 41(22), 1213 - 1214. https://doi.org/10.1049/el:20052799

8. Pfister, A. (1992). Novel CMOS Schmitt trigger with controllable hysteresis. Electron. Lette., 28, 639-641. https://doi.org/10.1049/el:19920404

9. Saini, S., Veeramachaneni, S., Kumar, A. M. \& Srinivas, M.B. (2009). Schmitt trigger as an alternative to buffer insertion for delay and power reduction in VLSI interconnects. TENCON2009-2009 IEEE Region10 conferences 1 -5. https://doi.org/10.1109/TENCON.2009.5396104

10. Saxena, A., Shrivastava, A. \& Akashe, S. (2014). Design and performance evaluation of Schmitt trigger for nano scale CMOS. American scientific publishers, Quantum Matter 3 (1-4). https://doi.org/10.1166/qm.2014.1095

11. Steyaert, M. \& Sansen, W. (1986). Novel CMOS Schmitt trigger. IEEE Transication. Electron Devices, 22(4), 203 - 204. https://doi.org/10.1049/el:19860142

12. Wang, Z. (1991). CMOS adjustable Schmitt triggers. IEEE Transaction, Instrumentation, Measurement. 40(3), 601-605. https://doi.org/10.1049/el:19920404

13. Weste, N. \& Eshraghian, K. (1993). Principles of CMOS VLSI Design (A Systems Perspective), 2nd ed. Reading, MA: Addison Wesley. 